\title{
Comparison of capillary, venous and buffy coat blood samples in detecting Plasmodium species among malaria suspected patients attending at Hamusite health center. A cross-sectional study
}

Getu Abeje ${ }^{1}$, Woyneshet Gelaye ${ }^{2}$ and Getaneh Alemu ${ }^{2^{*}}$ (D)

\begin{abstract}
Background: Both capillary and venous blood samples have been interchangeably used for the diagnosis of malaria in Ethiopia. However, Plasmodium parasites are thought to be more concentrated in capillary than in venous blood. Hence, selecting a sample source where parasites are more concentrated is indispensable approach in order to maximize the accuracy of blood film microscopy. Therefore, the present study aimed to compare the detection rate and the parasitemia level of Plasmodium species from conventional capillary and venous blood films, and buffy coat preparations.
\end{abstract}

Methods: A facility based cross-sectional study was conducted from Feburary to March 2020 among 210 febrile patients attending Hamusite health center, northwest Ethiopia. Capillary and venous blood samples were collected and buffy coat was prepared from each sample. Thin and thick blood films were prepared, stained, and examined microscopically following standard protocol. Data were analysed using Statistical Package for Social Sciences Software version 20 and Med-Calc software version 19.3.

Results: Capillary blood buffy coat $(61 / 210,29.0 \%)$ had significantly higher detection rate as compared to capillary $(48 / 210,22.9 \%)$ and venous $(42 / 210,20.0 \%)$ blood films $(p<0.001)$. However, no significant difference was observed between capillary and venous blood films $(p=0.070)$ in detecting Plasmodium species. The highest and the lowest mean asexual stage parasite counts were found in capillary blood buffy coat (4692.88) and venous blood (631.43) films, respectively showing significant variations $(p<0.001)$. Mean gametocyte count was also highest in capillary blood buffy coat (3958.44). As compared to capillary blood buffy coat, the sensitivity of venous blood buffy coat, capillary blood film and venous blood film were 73.8, 78.7, 68.9\%, respectively.

Conclusion: Capillary blood buffy coat samples showed the highest sensitivity in detecting and quantitating malaria parasites that its use should be promoted in clinical settings. However, conventional capillary and venous blood films could be used interchangeably.

Keywords: Capillary blood, Venous blood, Buffy coat, Malaria, Detection rate

\footnotetext{
* Correspondence: getanehmlt@gmail.com

${ }^{2}$ Department of Medical Laboratory Science, Bahir Dar University, Bahir Dar, Ethiopia

Full list of author information is available at the end of the article
}

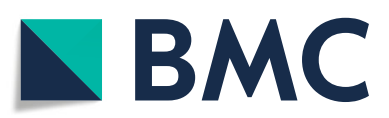

(- The Author(s). 2021 Open Access This article is licensed under a Creative Commons Attribution 4.0 International License, which permits use, sharing, adaptation, distribution and reproduction in any medium or format, as long as you give appropriate credit to the original author(s) and the source, provide a link to the Creative Commons licence, and indicate if changes were made. The images or other third party material in this article are included in the article's Creative Commons licence, unless indicated otherwise in a credit line to the material. If material is not included in the article's Creative Commons licence and your intended use is not permitted by statutory regulation or exceeds the permitted use, you will need to obtain permission directly from the copyright holder. To view a copy of this licence, visit http://creativecommons.org/licenses/by/4.0/ The Creative Commons Public Domain Dedication waiver (http://creativecommons.org/publicdomain/zero/1.0/) applies to the data made available in this article, unless otherwise stated in a credit line to the data. 


\section{Background}

Malaria is a vector-borne disease caused by protozoan parasites of the genus Plasmodium. Four Plasmodium species; namely Plasmodium( $P$ ) falciparum, $P$. vivax, $P$. malaria and $P$. ovale commonly infect humans. A fifth species, $P$. knowlesi, has recently been reported to infect humans in Southeast Asia $[1,2]$. Plasmodium falciparum and $P$. vivax are the most prevalent species in the world, and the former is the most virulent species in terms of both morbidity and mortality [3, 4].

Malaria is a global public health problem as it remains one of the most important causes of morbidity and mortality throughout the world [5]. However, most (90\%) of the malaria cases occur in the African Region [6]. In 2018, about 228 million cases were reported globally with 405 thousand deaths with $67 \%$ of the deaths occured among under 5 years-old children, the most vulnerable groups. About 93\% of the deaths were in Africa [5]. In Ethiopia, approximately $75 \%$ of the land mass, where $68 \%$ of the population lives, is malarious [7]. Despite considerable national efforts being made to reduce morbidity and mortality due to malaria in Ethiopia, the disease is still a prominent public health problem throughout the country [8].

Humans are primarily infected through the bite of an infected female Anopheles mosquito that inoculates sporozoites during blood meal [9]. After inoculation, parasites circulate with blood and those reaching to the liver undergo one cycle development. Then after, parasites infect and multiply inside red blood cells (RBCs) to bring the characteristic signs and symptoms [10]. The clinical manifestations usually appear within 10 to 15 days after the mosquito bite and includes fever, headache and vomiting. If not treated, malaria can become lifethreatening by disrupting blood supply to vital organs [11].

In order to reduce the burden and public health impact of malaria, early diagnosis and prompt treatment of patients are key strategies. The diagnosis is made using different techniqes ranging from the simple rapid diagnostic tests (RDTs) to complex molecular methods. In routine diagnosis, microscopic examination of blood films (BFs) and multi-species RDTs are performed. According to the Ethiopian national malaria diagnosis and treatment guidline, all febrile patients visiting health posts should be tested using RDTs and patients visiting health centers and higher health institutions should be diagnosed with BF microscopy before prescribing antimalarial drugs [7].

The detection of Plasmodium parasites via light microscopy either from capillary or venous blood is still the primary method in most health care facilities equiped with clinical laboratory [12]. According to the World Health Organization guideline, blood smears can be prepared either from capillary or venous blood samples [13]. On the other side, malaria parasites are thought to be more concentrated in peripheral capillary blood than in venous blood, making capillary blood more sensitive. In line with this, a few previous studies have concluded that capillary blood is more sensitive for the detection of Plasmodium species than venous blood $[14,15]$. On the contrary, some other studies showed that there is no difference in both detection and parasitemia level among capillary and venous blood samples $[16,17]$. Hence, more data from different endemicity settings is required in order to solve the discrepancy and influence policy makers so that more sensitive samples will be recommended for examination.

In addition, Ethiopia is moving from malaria morbidity control to elimination [18]. At this stage, occurrence of light infection with low parasitemia level is expected in endemic areas of the country. This, in turn, calls for use of more sensitive diagnostic techniques capable of detecting such light infections. Molecular techniques like polymerase chain reaction (PCR) are primary choices for this. However, PCR needs well trained personnel, complex laboratory, long time for result generation and it is costy. Hence, it is not feasible to apply PCR as a routine diagnostic technique in resource limited countries like Ethiopia. Maximizing the detection rate of blood film microscopy is an important alternative to tackle the existing problem. As part of this, collecting blood at the site where parasites are more concentrated or concentrating blood after collection (like buffy coat) should be promoted. Despite this, there is lack of data about variations in sensitivity of blood samples collected from capillary and venous sources as well as buffy coat (BC) concentrate in Ethiopia. Hence, the aim of the present study was to compare the detection rate of Plasmodium species from capillary, venous and buffy coat BFs among malaria suspected patients in Dera district, one of the known malarious areas in Amhara Region of Ethiopia.

\section{Methods}

\section{Study design and area}

A facility based cross-sectional study was conducted at Hamusite Health center, northwest Ethiopia from Feburary to March 2020. The health center serves for the people from Dera district. The district is located at geographic coordinates of $11^{\circ} 43^{\prime} 0^{\prime \prime}$ North and $37^{\circ} 38^{\prime} 0^{\prime \prime}$ East. The area is with an average altitude of $2077 \mathrm{~m}$ above sea level, mean annual rain fall of $1300 \mathrm{~mm}$ and mean annual temperature of $26^{\circ} \mathrm{C}$ (source: District health office).

\section{Sample size calculation and sampling technique}

This study was part of a project investigating 'Epidemiology and associated factors of malaria in Dera district'. 
Prevalence of Plasmodium infection was considered to calculate sample size using the single population proportion formula. Previous prevalence (p) of $8.7 \%$ in a similar geographical setting [19], 4\% margin of error (d) at 95\% confidence level $(Z \alpha / 2=1.96)$ and $10 \%$ of the initial sample size (19 participants) were assumed during sample size calculation. Hence, the final sample size was 210. Febrile patients who visited the health center laboratory for BF examination and met the eligibility criteria were recruited by systematic random sampling technique with sampling interval of 2 . Febrile patients clinically suspected of malaria who were $\geq 5$ years-old and volunteer to participate were included in the study. Individuals who took anti-malaria and antibiotics therapy with in four weeks before enrollment were excluded.

\section{Blood sample collection and processing}

Two milliliters of venous blood was collected from the cubital vein and capillary blood was collected by finger puncture. Venous and capillary blood samples were collected from each participant simultaneously with time gap of less than $5 \mathrm{~min}$. Thin and thick BFs were prepared using approximately $2 \mu \mathrm{l}$ and $6 \mu \mathrm{l}$ of blood, respectively. Furthermore, BC was prepared from both venous and capillary blood samples using heparnarized microhematocrit capillary tube. The capillary tube containing blood was centrifuged at $5000 \mathrm{rpm}$ for $5 \mathrm{~min}$. Then, the capillary tube was cut and the red cell layer just below the plasma (approximately 1-2 m meter) was aspirated using a micropipette and used for thin and thick BF preparatin. In this way, four BFs (from capillary blood, venous blood, capillary blood $\mathrm{BC}$ and venous blood $\mathrm{BC}$ ) were simultaneously prepared from each participant and stained with $10 \%$ giemsa for $10 \mathrm{~min}$ and then examined microscopically following standard protocol. Asexual parasite stages and gamatocytes were counted separately from each positive smear against 200 and 1000 WBCs, repectively [20]. For quantitative report, slides from each sample source were read twice and the mean parasite count was reported as the final parasite load.

In order to ensure data quality, standard operating procedures were strictly followed and quality of Giemsa staining reagent was checked every week using known positive and negative blood samples obtained from Amhara Public Health Institute (regional reference laboratory). Moreover, slides were read by two laboratory personnel where each reader was blind to results of the other. Both malaria microscopists are certified for 'malaria microscopy and quality assurance from the national medical parasitology reference laboratory' and had more than two years of practical experience at health institutions in malaria endemic areas. Discrepant results were examined by malaria laboratory experts from Amhara Public Health Institute.

\section{Statistical analysis}

Data were edited, entered, cleaned and then analyzed using Statistical Package for Social Science Software version 20. Descriptive statistics were manipulated to explain the study participants and to show the malaria prevalence and level of parasitemia from each BF preparation. Under nonparametric tests, 'related samples McNemar test' and 'related samples Wilcoxon signed rank test' were used to assess difference in parasite detection and parasitemia level, respectively between different BF preparations. 'T-test for paired samples' was used to calculate the mean difference in parasite count between capillary and venous blood sources. Sensitivity, specificity, Positive Predictive Value (PPV) and Negative Predictive Value (NPV) of capillary BF, venous BF and venous blood buffy coat results were calculated against that of capillary blood BC using Med-Calc software version 19.3. Kappa value (k) was also calculated to show the strength of test agreement between capillary BC results and other blood sources and preparations. Test agreements were declared as no agreement $(\mathrm{k} \leq 0)$, none to slight $(\mathrm{k}=0.01-0.2)$, fair $(\mathrm{k}=0.21-0.4)$, moderate $(\mathrm{k}=$ $0.41-0.60)$, substantial $(\mathrm{k}=0.61-0.80)$ and almost perfect $(\mathrm{k}=0.81-1.00)$ [21]. Differences in parasite detection rate and mean parasite count across different BF preparations were considered significant if $p$-value $\leq 0.05$ at 95\% confidence level.

\section{Results}

Socio-demographic characteristics of study participants Equal number of male and female participants, 105 each, were recruited in the study. Regarding to their age composition,153 (72.9\%) and 57 (27.1\%) participants were > 15 and $6-15$ years-old, respectively. One hundred ninety three (91.1\%) participants were rural residents while the remaining 17 (8.1\%) were urban dwellers.

\section{Detection of Plasmodium species and Parasitemia level}

Overall, 61 (29.0\%) participants were confirmed to be infected at least by a single species of Plasmodium at least by one sample source or BF preparation. Regarding to Plasmodium detection by sample source, 61 (29.0\%) and $45(21.4 \%)$ participants were positive from capillary and venous blood sources, respectively. Capillary blood BC preparation was the most sensitive sample being positive in 61 participants while conventional venous BF was the least sensitive where parasites were detected in 42 (20.0\%) participants (Table 1). The highest mean asexual stage parasite count was detected from capillary blood BC (4692.88 parasites/ $\mu$ l) while the lowest mean parasite count was from venous BF (2070.73 parasites $/ \mu \mathrm{l})$. 
Table 1 Prevalence of Plasmodium species from capillary and venous blood sources among malaria suspected patients attending Hamusite Health center,northwest Ethiopia from February to March 2020

\begin{tabular}{|c|c|c|c|c|}
\hline Variable & Total examined & Category & Number positive & Prevalence (in \%) \\
\hline \multirow[t]{4}{*}{ Cummulative } & 210 & Overall Plasmodium & 61 & 29.0 \\
\hline & & P. falciparum only & 41 & 19.5 \\
\hline & & P. vivax only & 10 & 4.8 \\
\hline & & Mixed infection & 10 & 4.8 \\
\hline \multirow[t]{2}{*}{ Blood Source } & 210 & Capillary & 61 & 29.0 \\
\hline & & Venous & 45 & 21.4 \\
\hline \multirow[t]{4}{*}{ Capillary BF } & 210 & Overall Plasmodium & 48 & 22.9 \\
\hline & & P. falciparum only & 29 & 13.8 \\
\hline & & P. vivax only & 9 & 4.3 \\
\hline & & Mixed infection & 10 & 4.8 \\
\hline \multirow[t]{4}{*}{ Venous BF } & 210 & Overall Plasmodium & 42 & 20.0 \\
\hline & & P. falciparum only & 23 & 11.0 \\
\hline & & P. vivax only & 9 & 4.3 \\
\hline & & Mixed infection & 10 & 4.8 \\
\hline \multirow[t]{4}{*}{ Capillary blood BC } & 210 & Overall Plasmodium & 61 & 29.0 \\
\hline & & P. falciparum only & 41 & 19.5 \\
\hline & & P. vivax only & 10 & 4.8 \\
\hline & & Mixed infection & 10 & 4.8 \\
\hline \multirow[t]{4}{*}{ Venous blood BC } & 210 & Overall Plasmodium & 45 & 21.4 \\
\hline & & P. falciparum only & 26 & 12.4 \\
\hline & & P. vivax only & 9 & 4.3 \\
\hline & & Mixed infection & 10 & 4.8 \\
\hline \multirow[t]{4}{*}{ Parasite stage detected } & 61 & Trophozoite only & 44 & 72.1 \\
\hline & & Schizont only & 1 & 1.6 \\
\hline & & Gametocyte only & 2 & 3.3 \\
\hline & & More than 1 stage & 14 & 23.0 \\
\hline \multirow[t]{5}{*}{ Gametocyte prevalence } & 210 & Over all & 16 & 7.6 \\
\hline & & Capillary BF & 15 & 7.1 \\
\hline & & Venous BF & 14 & 6.7 \\
\hline & & Capillary blood BC & 16 & 7.6 \\
\hline & & Venous blood BC & 15 & 7.1 \\
\hline
\end{tabular}

Key to abrreviations: $\mathrm{BF}=$ Blood Film, $\mathrm{BC}=$ Buffy Coat

Table 2 Mean parasite count from capillary and venous blood samples among malaria suspected patients attending Hamusite Health center, northwest Ethiopia from February to March 2020

\begin{tabular}{|c|c|c|c|c|c|c|}
\hline Parasite stages & Category & Number of cases & Minimum count & Maximum count & Mean count & SD \\
\hline \multirow[t]{4}{*}{ Asexual Stages } & Capillary BF & 47 & 80 & 26,000 & 2931.06 & 2320.00 \\
\hline & Venous BF & 41 & 40 & 20,000 & 2070.73 & 1776.05 \\
\hline & Capillary Blood BC & 59 & 40 & 30,000 & 4692.88 & 3217.24 \\
\hline & Venous Blood BC & 43 & 120 & 28,000 & 3728.37 & 2483.90 \\
\hline \multirow[t]{4}{*}{ Gametocyte } & Capillary BF & 15 & 200 & 4000 & 1177.33 & 512.62 \\
\hline & Venous BF & 14 & 240 & 1680 & 631.43 & 228.87 \\
\hline & Capillary Blood BC & 16 & 600 & 20,000 & 3958.44 & 2314.25 \\
\hline & Venous Blood BC & 15 & 400 & 12,000 & 2096.67 & 1495.39 \\
\hline
\end{tabular}


Similarly, the highest gametocyte count was observed in capillary blood BC (3958.44 gametocytes/ $\mu \mathrm{l})$ as opposed to only 631.43 gametocytes/ $\mu$ from venous BF (Table 2 ).

\section{Comparison of parasite detection across capillary and venous samples}

Detection of Plasmodium species in capillary BC was significantly higher as compared to that of capillary and venous $\mathrm{BF}(p<0.001)$. However, the difference in detection rate of parasites in capillary and venous $\mathrm{BF}$ was not statistically significant $(p=0.070)$ (Table 3$)$. The mean asexual parasite count significantly varies across different sample sources and smear preparations. Similarly, mean gametocyte count between capillary blood $\mathrm{BC}$ and capillary $\mathrm{BF}(p=0.032)$, capillary blood $\mathrm{BC}$ and venous blood $\mathrm{BC}(p=0.005)$, capillary $\mathrm{BF}$ and venous $\mathrm{BF}(p=0.041)$ were also significantly different (Table 4 ). We planned to compare the performance of each preparation using the combined result as a reference. However, we found that the detection rate of capillary blood $\mathrm{BC}$ was the same as that of the combined result. Hence, we used findings of capillary blood $\mathrm{BC}$ as a reference to evaluate the performance of the other blood film preparations. Accordingly capillary $\mathrm{BF}$, venous $\mathrm{BF}$ and venous blood $\mathrm{BC}$ were with sensitivity of $78.7,68.9$ and $73.8 \%$, respectively in detecting Plasmodium infection as compared to capillary blood BC results. The NPV of mentioned preparations were $92.0,88.7$ and $90.3 \%$, respectively while the specificity and PPV were $100 \%$ for all preparations. However capillary BF,venous blood BC and venous BF had substantial to almost perfect test agreement with that of capillary BC result (kappa values of $0.84,0.76$ and 0.80 , respectively). Species level analysis also showed that capillary $\mathrm{BF}$, venous $\mathrm{BF}$ and venous blood $\mathrm{BC}$ had low sensitivity $(70.7,56.1$ and $63.4 \%$, respectively) in detecting P. falciparum mono-infection but higher sensitivity $(90 \%$ for each preparation) in detecting $P$. vivax monoinfection. There was $100 \%$ agreement across all samples in detecting mixed infection (Table 5).

\section{Discussion}

Malaria is still a puplic health concern in Ethiopia even though substantial decrease in malaria related mortality and morbidity due to large scale intverentions $[8,18]$. Early and accurate case detection is essential in the fight against the disease which, in turn, requires examination of the right clinical sample using a sensitive diagnostic method. In the present study, we have compared the sensitivity of venous and capillary BFs as well as BC preparations from both sample sources for the detection and quantitation of Plasmodium species.

Over all parasite detection was higher from capillary blood than venous blood ( $29.0 \%$ vs $21.4 \%$ ) which goes in line with findings from Cameroon where capillary and venous BFs detected parasites in 29.3 and $17.3 \%$ of the participants, respectively $(p=0.0109)$ [14]. The possible reason for the difference might be due to the ability of $P$. falciparum (the most prevalen species in Ethiopia and in the present study) infected RBCs to sequester in the endothelial cells lining capillary blood vessles. Hence, during finger pricking, the microvasculature where sequesterd parasites are located are direcly accessed [22]. On the contrary, this phenomenon will limit presence or abandence of parasites in the venous blood. Moreover, presence of parasite stages in peripheral blood greatly varies by time, especially in venous blood [3] that repeatedly collected samples are required before ruling out the infection in each of the sample sources [14]. However, in the present study, blood was collected only once due to logistic problems.

More specifically, the detection rate was much higher in BC concentrates of capillary blood. This is justifyable because parasitized RBCs easily float and concentrate in or slightly below the $\mathrm{BC}$ upon centrifugation [22]. This characteristic resulted higher parasitaemia levels in specimens prepared from $\mathrm{BC}$ samples than respective conventional BFs as supported by results of the present study. The present finding is in line with previous study results which showed $100 \%$ sensitivity of BC preparations [23, 24]. The difference in Plasmodium detection between conventional capillary and venous BFs was not significant $(p=0.070)$ in the present study, pronouncing the importance of $\mathrm{BC}$ preparation. However, the mean capillary BF parasitemia level was significantly higher than that of venous $\mathrm{BF}(p<0.001)$ which was in line with findings from Gabon [15]. This result contradicts with reports from Cameroon [17, 20], Burkina Faso [25] and Ugand [16] where no significant difference in mean parasitemia level between capillary and venous BF examinations was noted. This might be due to the difference

Table 3 Difference in detecting Plasmodium species across different sample sources and preparations among malaria suspected patients attending Hamusite Health center, northwest Ethiopia from February to March 2020

\begin{tabular}{lll}
\hline Comparison & & \\
\hline Difference in detecting Plasmodium species between & Capillary BF and venous BF & 0.070 \\
& Capillary BF and capillary BC & 0.000 \\
& Capillary BF and venous BC & 0.453 \\
& Capillary BC and venous BF & 0.000 \\
\hline
\end{tabular}


Table 4 Mean difference in parasite count from capillary and venous blood samples among malaria suspecte patients attending Hamusite Health center, northwest Ethiopia from February to March 2020

\begin{tabular}{|c|c|c|c|c|c|c|}
\hline Parasite stages & Comparison & MD & SD & SE & $95 \% \mathrm{Cl}$ of $\mathrm{MD}$ & P-value \\
\hline \multirow[t]{5}{*}{ Asexual stages } & Capillary Blood BC vs Capillary BF & 2819.57 & 2659.08 & 775.73 & $1258.11-4381.04$ & 0.001 \\
\hline & Capillary Blood BC vs venous BF & 4354.63 & 2863.39 & 894.37 & $2547.04-6162.23$ & 0.000 \\
\hline & Capillary Blood BC vs venous Blood BC & 2478.14 & 2227.82 & 679.48 & $1106.90-3849.38$ & 0.001 \\
\hline & Capillary BF vs venous BF & 1277.50 & 870.4 & 275.25 & 720.77-1834.24 & 0.000 \\
\hline & venous Blood $B C$ vs venous $B F$ & 1832.68 & 1559.83 & 487.21 & $847.10-2817.37$ & 0.001 \\
\hline \multirow[t]{5}{*}{ Gametocytes } & Capillary Blood BC vs Capillary BF & 2978.33 & 2418.87 & 1249.10 & $299.29-5657.38$ & 0.032 \\
\hline & Capillary Blood BC vs venous BF & 2695.00 & 2788.2 & 1546.62 & $-674.79-6064.79$ & 0.107 \\
\hline & Capillary Blood BC vs venous BC & 2059.00 & 1181.37 & 610.06 & $750.56-3367.44$ & 0.005 \\
\hline & Capillary BF vs venous BF & 615.71 & 509.27 & 272.22 & $27.63-1203.80$ & 0.041 \\
\hline & venous $B l o o d B C$ vs venous $B F$ & 1586.43 & 1582.07 & 845.65 & $-240.49-3413.35$ & 0.083 \\
\hline
\end{tabular}

Key to abbrevations: $M D$ mean difference; $S E$ standard error of the mean; $S D$ standard deviation, $C I$ confidence interval

in sample size, population recruited, method of BF preparations and examination, and time of blood collection. For example, the study from Cameroon [17] collected data from asymptomatic children.

The sensitivity of capillary $(78.7 \%)$ and venous $(68.9 \%)$ BFs observed in the present finding was comparable to results reported from Gabon which was 81.5 and $73.4 \%$, respectively [15]. A sensitivity gap of $9.8 \%$ observed between capillary and venous BF in the present study was also comparable with the sensitivity gap of $12 \%$ reported from Cameroon [14]. Such differences might be due to the inherent ability of $P$. falciparum infected RBCs to contain highly variable parasite proteins on their surface, which have the ability to express in capillary blood vessels leading higher parasitemia in capillary blood compared to venous blood. Moreover, infected erythrocytes that do not adhere to vascular endothelium cells have probability to become eliminated in the spleen which leads to lower parasitemia, and hence lower detection, in venous blood [26].

The sensitivity gap of $14.6 \%$ between capillary and venous $B F$ in detecting $P$. falciparum mono-infection in the present study was slightly lower than results from a study conducted in Gabon with gap of $17.9 \%$ [15]. On the other hand, no such difference in sensitivity of capillary and venous BF was observed in detecting P.vivax mono-infection and mixed infections. There was almost perfect test agreement between the blood sources in detecting P.vivax and mixed-infection. Plasmodium vivax doesn't have the characteristic to sequester in capillary vessels making no significant difference in parasite abundence between capillary and venous samples.

Capillary BF,venous BC and venous BF had sensitivity of 93.8, 99.5 and 93.8\%; and NPV of 99.5, 87.5 and 99.0\%, respectively in detecting Plasmodium gametocytes as compared to capillary $\mathrm{BC}$ with almost perfect

Table 5 Performance of capillary blood film, venous blood film and venous blood buffy coat samples in detecting Plasmodium species among malaria suspected patients attending Hamusite Health center, northwest Ethiopia from February to March 2020

\begin{tabular}{|c|c|c|c|c|c|c|}
\hline Parameter & Sample & Sensitivity & Specificity & PPV & NPV & Kappa value \\
\hline \multirow[t]{3}{*}{ In detection of overall Plasmodium infection } & Capillary BF & 78.7 & 100 & 100 & 92.0 & 0.84 \\
\hline & Venous BF & 68.9 & 100 & 100 & 88.7 & 0.76 \\
\hline & Venous blood BC & 73.8 & 100 & 100 & 90.3 & 0.80 \\
\hline \multirow[t]{3}{*}{ In detection of $P$. falciparum mono-infection } & Capillary BF & 70.7 & 100 & 100 & 93.4 & 0.795 \\
\hline & Venous BF & 56.1 & 100 & 100 & 90.4 & 0.673 \\
\hline & Venous Blood BC & 63.4 & 100 & 100 & 91.8 & 0.736 \\
\hline \multirow[t]{3}{*}{ In detection of $P$. vivax mono-infection } & Capillary BF & 90 & 100 & 100 & 99.5 & 0.945 \\
\hline & Venous BF & 90 & 100 & 100 & 99.5 & 0.945 \\
\hline & Venous Blood BC & 90 & 100 & 100 & 99.5 & 0.945 \\
\hline \multirow[t]{3}{*}{ In detection of gametocytes } & Capillary BF & 93.8 & 100 & 100 & 99.5 & 0.965 \\
\hline & Venous BF & 87.5 & 100 & 100 & 99.0 & 0.928 \\
\hline & Venous Blood BC & 93.8 & 100 & 100 & 99.5 & 0.965 \\
\hline
\end{tabular}


test agreement $(k>0.80)$. Conventional capillary BF had more gametocyte parasite count than that of venous $\mathrm{BF}$ in the present study $(p=0.041)$. This difference might be due to the reason that capillary blood is utilized by the Anopheles mosquito and gametocytes are parasite stages infecting the insect vectors during blood meals. So, in order to perpetuate the life cycle, gametocytes might have higher tendency to be abundant in capillary blood. This was similiar with the study result in Gabon and Uganda where capillary BF had higher gametocyte count than venous $B F[15,16]$.

In addition, the sensitivity of capillary blood BC $(100 \%)$ in the present study was higher than a sensitivity of $27.8 \%$ reported from Bankgkok [27]. On the contrary, the sensitivity of venous blood BC $(73.8 \%)$ observed in the present study was lower than $95.8 \%$ sensitivity of venous blood BC reported from Cameron [22] and 100, 96 and $92.7 \%$ reported from three separate studies in India [28-30]. The reason for this difference might be due to differences in $\mathrm{BC}$ preparation procedure. We prepared $\mathrm{BC}$ in heparinized capillary tube by centrifuging for 5 min while separation of $\mathrm{BC}$ was made using wintrobe tube by centrifuging for $12-15 \mathrm{~min}$ in previous studies. In addition,we have used BC thick and thin films examined under 100x magnification with the aid of bright field microscope. In studies from India, quantitative $\mathrm{BC}$ preparation tube was used, and after centerifuging with high speed $(12,000 \mathrm{rpm})$, it was examined with the aid of fluorescent microscope which increases the detection of Plasmodium species [26-28]. Similary sample size, local malaria prevalence and parasitemia level might affect the sensitivity. Further more, similar higher sensitivity results were reported from Thandalam [31]. In general, the present study showed that false negative results were higher in venous blood sample (9\%) than in capillary blood sample with a sensitivity and parasitemia level gap of $31.1 \%$ and 2622.11 , respectively. This finding facilitates use of capillary blood sample than venous blood sample not to miss light infections. The present study had limitations that we didn't include asymptomatic study participants where light infections are expected. Moreover, performance of capillary and venous blood samples in detecting malaria parasites was evaluated using BF microscopy. Use of more sensitive molecular methods (like polymerase chain reaction) would help to give definitive conclusions.

\section{Conclusions}

Use of capillary blood samples produces more accurate results than using venous blood samples in detecting malaria parasites. Capillary blood BC samples showed greater sensitivity in detecting $P$. falciparum monoinfection and in determing parasitemia level as compared to all other sample sources. Therefore, use of capillary blood BC should be promoted in clinical settings. We also recommend further studies assessing the sensitivity of capillary and venous samples in detecting Plasmodium species among asymptomatic individuals and by using more sensitive molecular techmiques.

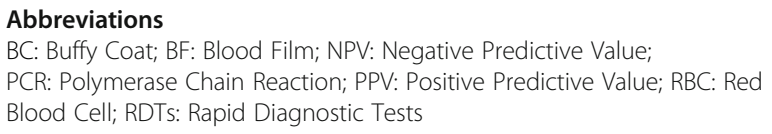

\section{Acknowledgments}

We thank ethical review comittee of Bahir Dar University, College of Medicine and Health Sciences for giving ethical approval. We,also thank Amhara Public Health Institute, South Gonder Zonal Health Department, Dera Woreda Health Office, and Hamusite Health center for giving permision and their support during data collection. Our regards also go to the study participants for participating in the study.

\section{Authors' contributions}

GA1: undertook the data collection, slide reading and participated in data analysis and manuscript preparation. WG: participated in data analysis and write up of the manuscript. GA2: conceived the study, participated in slide reading, data analysis and write up of the manuscript. All authors read and approved the final version of the manuscript.

\section{Funding}

Bahir Dar University funded for data collection. No fund was obtained for data analysis and manuscript preparation.

\section{Availability of data and materials}

The original data for this study is available from the corresponding author.

\section{Declarations}

\section{Ethics approval and consent to participate}

Ethical approval was obtained from Bahir Dar University, College of Medicine and Health Sciences Ethical Review Committee with project code of 008/ 2020 prior to the commencement of the study. Permission letter was obtained from Amhara Public Health Institute and Support letter was also obtained from South Gonder Zonal Department, Dera district Health Office and Hamusite Health Center. Informed written consent was obtained from each study participant with age 16 and above. Written informed consent was obtained from parents or guardians for participants with age below 16 Blood film microscopy results were promptly communicated to attending clinicians for appropriate treatmet.

Consent for publication

Not applicable.

\section{Competing interests}

The authors declare that they have no competing interests.

\section{Author details}

${ }^{1}$ Department of Biomedcal Science, Samara University, Samara, Ethiopia. 2Department of Medical Laboratory Science, Bahir Dar University, Bahir Dar, Ethiopia.

Received: 27 October 2020 Accepted: 4 June 2021

Published online: 15 June 2021

References

1. Collins WE. Plasmodium knowlesi: a malaria parasite of monkeys and humans. Annual Revolution Entomol. 2012;57(1):107-21. https://doi.org/1 0.1146/annurev-ento-121510-133540.

2. World Health Organization. Fact Sheet about malaria. Available at www. who.int/news-room/fact-sheets/detail/malaria. Accessed on 10 December 2019

3. Ferri F. Protozoal infections. Ferri's Color Atlas and Text of Clinical Medicine. Elsevier Health Sciences, Chapter 332; 2009. 
4. World Health Organization. Guidelines for the treatment of malaria: available at www.who.int/malaria/publications/atoz/9789241549127. Accessed on 15 December 2019.

5. World Health Organization. World Malaria Report 2017c. Available at https:// www.who.int/malaria/publications/world-malaria-report-2017c. Accessed on 15 December 2019.

6. World Health Organization. World Malaria Report 2017b. Available at https:// www.who.int/malaria/publications/world-malaria-report-2017b. Accessed on 16 December 2019.

7. Federal Ministry of Health. National five-year strategic plan for malaria prevention and control in Ethiopia 2006-2010. Addis Ababa; 2010.

8. World Health Organization. World malaria report 2019. Available at www. who.int/malaria/publications/world-malaria-report-2019/en. Accessed on 23 July 2020.

9. Kayser. Medical Microbiology, part V (Parasitology). 2nd ed; 2005. p. p520.

10. Antia M, Herricks T, Rathod K. Microfluidic modeling of cell- cell interactions in malaria pathogenesis. PLoS Pathogens. 2007;3(7):e99 Doi :10.1371/journal. Ppat. 0030099.

11. Clark I, Cowden W. The pathophysiology of Plasmodium falciparum malaria. Pharmacol Therapeutics. 2003;99(2):221-60. https://doi.org/10.1016/s016372 58(03)00060-3

12. Mukadi P. External quality assessment of malaria microscopy in the Democratic Republic of the Congo. Malar J. 2011;10(1):308. https://doi.org/1 0.1186/1475-2875-10-308.

13. World Health Organization. Guidelines for the treatment of malaria, 3rd ed. World Health Organization, Geneva,Switzerland. Available at http://www. who.int/malaria/publications/atoz/9789241549127/en/. Accessed on 18 March 2017

14. Njundaa N, Nsaghab F, Kamgaa M, Mokenyua KE. Comparison of capillary and venous blood using blood film microscopy in the detection of malaria parasites: a hospital based study. Sci J Microbiol. 2013;2(5):89-94.

15. Mischlinger J, Veletzky L, Groger M, Zoleko R. AdegnikaA, Agnandji ST., et al. use of capillary bloodsamples leads to higher Parasitemia estimates and higher diagnosticsensitivity of microscopic and molecular diagnostics of malaria than venousblood samples. J Infect Dis. 2018;218(8):1296-305. https://doi.org/10.1093/infdis/jiy319.

16. Lehane A, Moses W, Martina W, Musleehat H, Megan C, Sylvia K, et al. Comparison on simultaneous capillary andvenous parasite density and genotypingresults from children and adults with uncomplicated malaria. BMC Infect Dis. 2019:19(1):559. https://doi.org/10.1186/s12879-019-4174-1.

17. Sandeu MM, Tchioffo MT, Abate L, Gimonneau G, Awono A, Nsango SE, et al. Do the venousblood samples replicate malaria parasite densities found in capillary blood a field study performed in naturally-infected asymptomatic children in Cameroon. Malar J. 2017;16(1):345. https://doi. org/10.1186/s12936-017-1978-6

18. President's malaria initiative Ethiopia. Malaria operational plan FY 2019.

19. Biniyam B. A four year trend analysis of malaria prevalence in aysaita primary hospital, aysaita woreda, afar regional state, northeast Ethiopia (Doctoral dissertation, Addis Ababa Universty). Accessed 7 December, 2019.

20. Chotivanich K, Silamut K, Day NPJ. Laboratory diagnosis of malaria infectiona short review of methods. Aust J Med Sci. 2006;27:11-5.

21. Landis JR, Kock GG. The measurement of observer agreement for categorical data. Biometrics. 1977;33(1):159-74. https://doi.org/10.2307/252 9310.

22. Anna D, Longdoh S, Njunda N, Jules C, Nguedia A. Comparing the Buffy Coat and Traditional Blood Smears in the MicroscopicDiagnosis of Malaria in Cameroon. Int J Malaria Res Rev www.resjournals,org/IJMRISSN. 2014;2(2): 2346-7266.

23. Bandari PL, Rajeev A, Bhandari P. Comparative study of peripherial blood smear . quantitative buffy coat and modified centrifuged blood smear in malaria diagnosis. Indian J Pathol. 2008;51:108-12.

24. Duangdee C, Tangpukdee N, Krudsood S, Wilairatana P. Use of buffy coat thick films in detecting malaria parasites in patients with negative conventional thick films. Asian Pac J Trop Biomed. 2012;2(4):301-3. https:// doi.org/10.1016/S2221-1691(12)60027-6.

25. Ouedraogo JB, Gbary AR. Etude Comparative De La Densite Parasitaire De Plasmodium Falciparum Dans e Sang Capillaire Et Dans e Sang Veineu Che Des Porteurs Asmptomati uese gion de obo-Dioulasso, Burkinfaso. Eedecine d'Afrique Noire, vol. 38; 1991.
26. Janeway CA Jr, Travers P, Walport M, et al. Immunobiology: The Immune System in Health and Disease. 5th edition. New York: Garland Science; 2001. Available from: https://www.ncbi.nlm.nih.gov/books/NBK10757/.

27. Davis $R$, Flanigan $T$, Wilson E. Passive gravitational sedimentation of peripheral blood increases the sensitivity of microscopic detection of malaria. Asian Pac J Trop Med. 2013;6(7):552-5. https://doi.org/10.1016/S1 995-7645(13)60095-4.

28. Pinto MJ, Rodrigues SR, Desouza R. Usefullness of quantitative buffy coat blood parasite detection systemin diagnosis of malaria in India. India J Med Microbiol. 2001;19(4):219-22.

29. Manali M, Kocharekar S, Debjani D. Comparative Study of Modified Quantitative Buffy Coatand Two Rapid Tests in Comparison with Peripheral BloodSmear in Malaria Diagnosis in Mumbai, India. J Parasitol Res. 2014;7: 194651.

30. Bhavnaben A. And Vora RComparison of diagnostic methods of malaria by peripheral smear, centrifuged buffy coat smear and rapid antigen detection test. Int J Res Med Sci. 2017;5(10):4532-7.

31. Dhinesh B, Jayakumar S, Kalyani MD, Renu M. Shameem. Correlation of quantitative Buffy coat, blood smear and antigen detection in diagnosing malarial InfectionThandalam. J Clin Diagn Res. 2011;5(5):961-3.

\section{Publisher's Note}

Springer Nature remains neutral with regard to jurisdictional claims in published maps and institutional affiliations.

\section{Ready to submit your research? Choose BMC and benefit from:}

- fast, convenient online submission

- thorough peer review by experienced researchers in your field

- rapid publication on acceptance

- support for research data, including large and complex data types

- gold Open Access which fosters wider collaboration and increased citations

- maximum visibility for your research: over $100 \mathrm{M}$ website views per year

At BMC, research is always in progress.

Learn more biomedcentral.com/submissions 